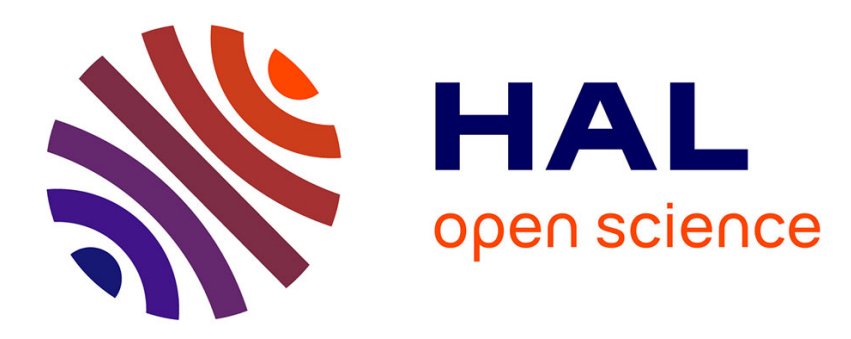

\title{
Vibration Testing for Detecting Internal Corrosion
}

Kaouthar Louati, Habib Ammari, Hyeonbae Kang, Eunjoo Kim, Hyundae Lee

\section{To cite this version:}

Kaouthar Louati, Habib Ammari, Hyeonbae Kang, Eunjoo Kim, Hyundae Lee. Vibration Testing for Detecting Internal Corrosion. 2007. hal-00125820

\section{HAL Id: hal-00125820 \\ https://hal.science/hal-00125820}

Preprint submitted on 22 Jan 2007

HAL is a multi-disciplinary open access archive for the deposit and dissemination of scientific research documents, whether they are published or not. The documents may come from teaching and research institutions in France or abroad, or from public or private research centers.
L'archive ouverte pluridisciplinaire HAL, est destinée au dépôt et à la diffusion de documents scientifiques de niveau recherche, publiés ou non, émanant des établissements d'enseignement et de recherche français ou étrangers, des laboratoires publics ou privés. 


\title{
Vibration Testing for Detecting Internal Corrosion*
}

\author{
Habib Ammari ${ }^{\dagger} \quad$ Hyeonbae Kang ${ }^{\ddagger} \quad$ Eunjoo Kim ${ }^{\ddagger} \quad$ Hyundae Lee ${ }^{\ddagger}$ \\ Kaouthar Louati ${ }^{\S}$
}

November 16, 2006

\begin{abstract}
The vibration behavior of structures can be characterized in terms of resonance frequencies and mode shapes which describe properties of the tested object in a global way but do not in general provide information about structural details. Following an asymptotic formalism, in much the same spirit as the work in [3] and recent text [1], we develop a simple method to address the inverse problem of identifying an internal corrosive part of small Hausdorff measure in a pipeline by vibration analysis. The viability of our reconstruction method is documented by a variety of numerical results from synthetic, noiseless and noisy data.
\end{abstract}

\section{Introduction}

Natural gas is supplied through a million miles of vast pipeline network. Pipeline companies have an impressive safety record due to the proactive role of standards and inspection of pipelines. Since the pipelines are getting old, there is a great need to identify corrosion, cracks, and other defects that can cause potential problems. Stress corrosion cracking can occur under a range of pipeline field conditions including soil type, stress, cathode potential, coating conditions and temperature. This type of defect is usually oriented along the lengthwise direction of the pipe. If not found and conditions persist, the cracks may grow and/or coalesce and eventually result in a leak or pipe rupture. There are also other types of defects that can occur in pipe structures. They are either critical to the safety of the pipeline like corrosion, welding cracks, pits, etc., or benign stringer-like internal inclusions. Non-destructive Inspection systems are strongly needed to be able to locate the defects early without false alarms from benign inclusions, and to characterize and size the defects for repair or replacement management.

*H.A. is partially supported by the Brain Pool Korea Program at Seoul National University, H.K. is partially supported by the grant KOSEF R01-2006-000-10002-0, E.K. and H.L. are supported by BK21 Math. division at Seoul National University.

${ }^{\dagger}$ Laboratoire Ondes et Acoustique, CNRS \& ESPCI, 10 rue Vauquelin, 75231 Paris Cedex 05, France (habib.ammari@polytechnique.fr).

${ }^{\ddagger}$ School of Mathematical Sciences, Seoul National University, Seoul 151-747, Korea (hkang@math.snu.ac.kr, kej@math.snu.ac.kr, hdlee@math.snu.ac.kr).

$\S$ Centre de Mathématiques Appliquées, Ecole Polytechnique, 91128 Palaiseau Cedex, France (louati@cmapx.polytechnique.fr). 
Many technologies have been developed for pipe inspections, but they are limited to detecting certain types of defects. Most of them are not suitable for detecting flaws or cracks in the axial direction. Recently, ultrasonic guided waves are being studied for the feasibility of detecting many kinds of defects that occur in pipes. One major benefit of guided waves is their rapid global inspection capabilities which enables them to inspect a structure line-by-line instead of point-by-point. However, defect classification and sizing by guided waves are still a major problem under investigation due to the complexity of the wave propagation characteristics.

In order to reduce complicated derivation in the analytic method, a simple two-dimensional model is adopted in this work to inversely determine the corrosive parts from the resonance frequencies and mode shapes.

Corrosion occurs in many different forms and several different models can be encountered in the literature (see, for example, Kaup and Santosa [12], Kaup et al. [13], Vogelius and $\mathrm{Xu}$ [16], Inglese [9], Luong and Santosa [10], Banks et al. [4] and references therein). In our model, the effect of corrosion is described by means of Robin boundary conditions. The study of a model like this is motivated by a number of favorable indications. A first indication is based on the observation that corrosion tends to roughen a surface: in fact, this effect can be modelled by the introduction of a thin coating characterized by rapid oscillations. In the limit where the thickness of the coating goes to zero and the rapidity of the oscillations diverges, the arising of Robin boundary conditions has been observed by Buttazo and Kohn [5]. On the other hand, the study of electrochemical corrosion processes can be based on Faraday's law which says that the mass loss which is a measure of corrosion is proportional to the normal current flux. In Vogelius and $\mathrm{Xu}$ [16] a potential model of this kind of process is proposed. If we linearize with respect to the transfer coefficient the nonlinear boundary conditions in [16], we get Robin boundary conditions.

In this work, we follow an asymptotic formalism, in much the same spirit as the work in [3] and recent text [1]. See also [7, 6, 14]. We derive asymptotic formulae for the effects of corrosion on resonance frequencies and mode shapes. Powerful techniques from the theory of meromorphic operator-valued functions and asymptotic analysis of integral kernels are combined for their rigorous proof. Based on these formulae we design a simple method for localizing the corrosion and estimating its extent.

Related works may be found in [15] and the references therein. Difficulties of this inverse problem result from its inherent ill-posedness and nonlinearity. Many authors have proposed various reconstruction algorithms, most of which are based on laborious least-square algorithms and Newton-type iteration schemes. In these methods, one must make a good initial guess. Without a good initial guess, one needs tremendous computational costs and time to get a close image to the true solution, since Newton-type iteration schemes may not converge to an approximate solution unless the initial guess is close to the true solution. Evidently, the success of Newton type procedures heavily depends on making a good initial guess. Unfortunately, the development of both the mathematical theory and the numerical algorithm for making a good initial guess seems to be in the early stages.

Our purpose in this work is to develop a simple method for determining the location of the corrosion and estimating its Hausdorff measure. From this information we may get an appropriate initial guess for the inverse problem .

Numerical examples are given in this paper in order to illustrate the main features of our approach for both asymptotically exact and noisy data. A systematic discussion however is not attempted, being left to further studies in specific situations of practical applicability. 
Our approach may be considered as a first step towards design of real-time, accurate and robust algorithms for corrosion detection from ultrasonic guided waves.

\section{Formal derivations}

To set up our inverse eigenvalue problem mathematically, we consider a simply connected bounded $\mathcal{C}^{2}$ domain $U$ in $\mathbb{R}^{2}$, and a simply connected $\mathcal{C}^{2}$ domain $D$ compactly contained in $U$. Let $\Omega=U \backslash \bar{D}$ represent the specimen to be inspected. We define $\Gamma_{e}=\partial U$ and $\Gamma_{i}=\partial D$ so that $\partial \Omega=\Gamma_{i} \cup \Gamma_{e}$. Suppose that the inaccessible surface $\Gamma_{i}$ contains a corrosive part $I$. The surface impedance (the corrosion coefficient) of $I$ is a positive constant $\gamma$. The domain $\Omega$ may be considered as a cross section of a pipe inside which there is a corrosive part. We assume that the one-dimensional Hausdorff measure $|I|$ of $I$ is small and denote it by $\epsilon$.

We now introduce the following functional spaces. Let $H^{1}(\Omega)$ denote the set of functions $w \in L^{2}(\Omega)$ such that $\nabla w \in L^{2}(\Omega)$. Let $H^{1 / 2}\left(\Gamma_{e}\right)$ be the set of traces of functions in $H^{1}(U)$. Further, we define $H^{2}(\Omega)$ as the space of functions $u \in H^{1}(\Omega)$ such that $\partial^{2} u \in L^{2}(\Omega)$ and the space $H^{3 / 2}(\Omega)$ as the interpolation space $\left[H^{1}(\Omega), H^{2}(\Omega)\right]_{1 / 2}$.

The eigenvalue problem in the presence of corrosion consists of finding $\omega_{\epsilon}>0$ such that there exists a nontrivial solution $v_{\epsilon}$ to

$$
\begin{cases}\left(\Delta+\omega_{\epsilon}^{2}\right) v_{\epsilon}=0 & \text { in } \Omega \\ -\frac{\partial v_{\epsilon}}{\partial \nu}+\gamma \chi(I) v_{\epsilon}=0 & \text { on } \Gamma_{i} \\ v_{\epsilon}=0 & \text { on } \Gamma_{e} \\ \int_{\Omega} v_{\epsilon}^{2}=1, & \end{cases}
$$

where $\nu$ is the outward unit normal to $D$ and $\chi(I)$ denotes the characteristic function on $I$. Throughout this paper the normal vector $\nu$ defined on either $\Gamma_{i}$ or $\Gamma_{e}$ is assumed to be directed outward to the relevant domain $D$ or $U$. So, it is directed inward to $\Omega$ on $\Gamma_{i}$.

It is well known that all eigenvalues of (2.1) are real, of finite multiplicity, have no finite accumulation points, and there are corresponding eigenfunctions which make up an orthonormal basis of $L^{2}(\Omega)$. See for example [11]. Let $\omega_{0}>0$ be for simplicity a simple eigenvalue for the Helmholtz equation in the absence of any corrosion. Let $v_{0}$ denote the corresponding eigenfunction, that is, the solution to

$$
\begin{cases}\left(\Delta+\omega_{0}^{2}\right) v_{0}=0 & \text { in } \Omega \\ -\frac{\partial v_{0}}{\partial \nu}=0 & \text { on } \Gamma_{i} \\ v_{0}=0 & \text { on } \Gamma_{e}\end{cases}
$$

such that $\int_{\Omega} v_{0}^{2}=1$.

The aim of this work is to detect the corrosive part $I$, in particular, its location $z \in I$ and its extend $\epsilon$, from variations of the modal parameters

$$
\left(\omega_{\epsilon}-\omega_{0},\left.\frac{\partial}{\partial \nu}\left(v_{\epsilon}-v_{0}\right)\right|_{\Gamma_{e}}\right) .
$$


We seek a solution of (2.1) for $\epsilon$ small, for which $\omega_{\epsilon} \rightarrow \omega_{0}$ as $\epsilon$ goes to zero. The expansion of $\omega_{\epsilon}$ must begin with $\omega_{0}$, and the expansion of $v_{\epsilon}$ must begin with $v_{0}$; so we write:

$$
\begin{aligned}
& \omega_{\epsilon}=\omega_{0}+\epsilon \omega_{1}+\epsilon^{2} \omega_{2}+\ldots, \\
& v_{\epsilon}=v_{0}+\epsilon v_{1}+\epsilon^{2} v_{2}+\ldots \text { in } \Omega,
\end{aligned}
$$

where $v_{1}, v_{2}, \ldots$ and $\omega_{1}, \omega_{2}, \ldots$ are to be found.

Now we substitute (2.4) into the Helmholtz equation (2.1) and equate terms of each power in $\epsilon$. This yields:

$$
\begin{cases}\left(\Delta+\omega_{0}^{2}\right) v_{1}=-2 \omega_{0} \omega_{1} v_{0} & \text { in } \Omega \\ \frac{\partial v_{1}}{\partial \nu}=\frac{1}{\epsilon} \chi(I) \gamma v_{0} & \text { on } \Gamma_{i} \\ v_{1}=0 & \text { on } \Gamma_{e}\end{cases}
$$

Observe that since $|I|=\epsilon, \frac{1}{\epsilon} \chi(I)$ is of order 1 . Since $\int_{\Omega} v_{\epsilon}^{2}=\int_{\Omega} v_{0}^{2}$, we also have an orthogonality condition:

$$
\int_{\Omega} v_{1} v_{0} d x=0
$$

Multiplying (2.5) by $v_{0}$ and integrating by parts yields that

$$
\begin{aligned}
2 \omega_{0} \omega_{1} & =-\int_{\Omega}\left(\Delta+\omega_{0}^{2}\right) v_{1} \cdot v_{0} d x \\
& =-\int_{\Gamma_{e}}\left(\frac{\partial v_{1}}{\partial \nu} v_{0}-v_{1} \frac{\partial v_{0}}{\partial \nu}\right)+\int_{\Gamma_{i}}\left(\frac{\partial v_{1}}{\partial \nu} v_{0}-v_{1} \frac{\partial v_{0}}{\partial \nu}\right) \\
& =\frac{\gamma}{\epsilon} \int_{I} v_{0}^{2} .
\end{aligned}
$$

So far we formally drove the following theorem, a rigorous proof of which will be given at the end of this paper.

Theorem 2.1 The following asymptotic expansion holds:

$$
\omega_{\epsilon}=\omega_{0}+\frac{\gamma}{2 \omega_{0}} \int_{I} v_{0}^{2}+O\left(\epsilon^{2}\right)
$$

as $\epsilon \rightarrow 0$. Furthermore,

$$
v_{\epsilon}=v_{0}+O(\epsilon)
$$

where $O(\epsilon)$ is in $H^{3 / 2}(\Omega)$ norm.

\section{Reconstruction method}

For $h \in H^{1 / 2}\left(\Gamma_{e}\right)$ such that $\int_{\Gamma_{e}} h \frac{\partial v_{0}}{\partial \nu}=0$, let $w_{h} \in H^{1}(\Omega)$ be the solution to

$$
\begin{cases}\left(\Delta+\omega_{0}^{2}\right) w_{h}=0 & \text { in } \Omega, \\ \frac{\partial w_{h}}{\partial \nu}=0 & \text { on } \Gamma_{i} \\ w_{h}=h & \text { on } \Gamma_{e} .\end{cases}
$$


Applying Green's formula, we obtain

$$
\gamma \int_{I} w_{h} v_{\epsilon}=\int_{\Gamma_{i}} w_{h} \frac{\partial v_{\epsilon}}{\partial \nu}=\int_{\Gamma_{e}} h \frac{\partial v_{\epsilon}}{\partial \nu}+\left(\omega_{\epsilon}^{2}-\omega_{0}^{2}\right) \int_{\Omega} v_{\epsilon} w_{h}
$$

Dividing (3.2) by $\omega_{\epsilon}^{2}-\omega_{0}^{2}$ and using (2.7) we induce

$$
\frac{\int_{I} w_{h} v_{\epsilon}}{\int_{I} v_{0}^{2}}=\frac{1}{\omega_{\epsilon}^{2}-\omega_{0}^{2}} \int_{\Gamma_{e}} h \frac{\partial v_{\epsilon}}{\partial \nu}+\int_{\Omega} v_{\epsilon} w_{h}+O(\epsilon) .
$$

By (2.8), we have

$$
\begin{aligned}
\int_{I} w_{h} v_{\epsilon} & =\int_{I} w_{h} v_{0}+O\left(\epsilon^{2}\right), \\
\int_{\Omega} v_{\epsilon} w_{h} & =\int_{\Omega} v_{0} w_{h}+O(\epsilon) .
\end{aligned}
$$

Therefore, we have

$$
\frac{w_{h}}{v_{0}}(z) \approx \frac{1}{2 \omega_{0}\left(\omega_{\epsilon}-\omega_{0}\right)} \int_{\Gamma_{e}} \frac{\partial v_{\epsilon}}{\partial \nu} h+\int_{\Omega} v_{0} w_{h} .
$$

This is the key observation on which our reconstruction procedure is based. Since we are in possession of $\omega_{\epsilon}-\omega_{0}$ and $\left.\frac{\partial v_{\epsilon}}{\partial \nu}\right|_{\Gamma_{e}}$, the reconstruction algorithm is as follows. Let $h=$ $h_{1}, h_{2}, \ldots, h_{n}$, where $\left\{h_{i}\right\}_{i=1}^{n}$ is a set of $n$ independent functions satisfying $\int_{\Gamma_{e}} h_{i} \frac{\partial v_{0}}{\partial \nu}=0$ for $i=1, \ldots, n$. For any $y \in \Gamma_{i}$ such that $v_{0}(y) \neq 0$ compute $\left(w_{h_{i}} / v_{0}\right)(y)$. The point $z$ can be found as the unique point where

$$
\frac{w_{h_{i}}}{v_{0}}(z)=\frac{1}{2 \omega_{0}\left(\omega_{\epsilon}-\omega_{0}\right)} \int_{\Gamma_{e}} \frac{\partial v_{\epsilon}}{\partial \nu} h_{i}+\int_{\Omega} v_{0} w_{h_{i}}, \quad \forall i=1, \ldots, n .
$$

The justification of our method is quite simple and natural. Let $\langle,\rangle_{\frac{1}{2},-\frac{1}{2}}$ denote the duality pair between $H^{1 / 2}\left(\Gamma_{i}\right)$ and $H^{-1 / 2}\left(\Gamma_{i}\right)$. Observe first that the following density result holds.

Lemma 3.1 If $\left\langle v_{0}, \phi\right\rangle=0$, then $\left\langle w_{h}, \phi\right\rangle=0$ for all $h \in H^{1 / 2}\left(\Gamma_{e}\right)$ such that $\int_{\Gamma_{e}} h \frac{\partial v_{0}}{\partial \nu}=0$ implies that $\phi=0$.

Proof. For $\phi \in H^{-1 / 2}\left(\Gamma_{i}\right)$ such that $\left\langle v_{0}, \phi\right\rangle=0$, let $u_{\phi}$ be the solution to

$$
\begin{cases}\left(\Delta+\omega_{0}^{2}\right) u_{\phi}=0 & \text { in } \Omega, \\ \frac{\partial u_{\phi}}{\partial \nu}=\phi & \text { on } \Gamma_{i} \\ u_{\phi}=0 & \text { on } \Gamma_{e}\end{cases}
$$

An integration by parts shows that $\int_{\Gamma_{e}} h \frac{\partial u_{\phi}}{\partial \nu}=0$ and therefore, by the unique continuation, $u_{\phi}=c v_{0}$ in $\Omega$, for some constant $c$. Thus, $\phi=0$, as desired. 
Suppose now that

$$
\frac{w_{h}}{v_{0}}(y)=\frac{1}{2 \omega_{0}\left(\omega_{\epsilon}-\omega_{0}\right)} \int_{\Gamma_{e}} \frac{\partial v_{\epsilon}}{\partial \nu} h+\int_{\Omega} v_{0} w_{h},
$$

for all $h \in H^{1 / 2}\left(\Gamma_{e}\right)$ such that $\int_{\Gamma_{e}} h \frac{\partial v_{0}}{\partial \nu}=0$. By integrating by parts and using Theorem 2.1 , we see that

$$
\int_{\Gamma_{i}} \frac{\partial v_{\epsilon}}{\partial \nu} w_{h} \approx-\epsilon \gamma v_{0}(y) w_{h}(y) \quad \text { on } \Gamma_{i}, \quad \forall h \in H^{1 / 2}\left(\Gamma_{e}\right) \text { such that } \int_{\Gamma_{e}} h \frac{\partial v_{0}}{\partial \nu}=0 .
$$

and therefore, by the density result in Lemma 3.1,

$$
\frac{\partial v_{\epsilon}}{\partial \nu} \approx-\epsilon \gamma v_{0}(y) \chi\left(I_{y}\right) \quad \text { on } \Gamma_{i}
$$

where $\left|I_{y}\right|=\epsilon$ and $y \in I_{y}$, from which (2.5) yields $y \approx z$. This shows that for $n$ large enough $z$ is uniquely determined by our algorithm.

Once $z$ is determined, the Hausdorff measure of the corrosive part can be estimated by

$$
\epsilon \approx \frac{2 \omega_{0}\left(\omega_{0}-\omega_{\epsilon}\right)}{\gamma v_{0}^{2}(z)}
$$

Note that we can not estimate $\epsilon$ separately from $\gamma$. We need to have an a prior knowledge of one of these two parameters in order to determine the other.

\section{Numerical Results}

This section presents results of some numerical experiments using the reconstruction method of the previous section. In the following, $U$ and $D$ are assumed to be the disks centered at the origin $(0,0)$, and of radii $r_{e}$ and $r_{i}$, respectively. We set $\Omega=U \backslash \bar{D}$, as before.

First we find the eigenvalue and eigenvector for (2.1) and (2.2). For convenience, using polar coordinates, we rewrite the equations in the following form:

$$
\begin{cases}\left(\frac{\partial^{2}}{\partial r^{2}}+\frac{1}{r} \frac{\partial}{\partial r}+\frac{1}{r^{2}} \frac{\partial^{2}}{\partial \theta^{2}}+\omega_{\epsilon}^{2}\right) v_{\epsilon}=0 & \text { in } \Omega:=[0,2 \pi] \times\left[r_{i}, r_{e}\right], \\ -\frac{\partial v_{\epsilon}}{\partial r}+\gamma \chi(I) v_{\epsilon}=0 & \text { on } \Gamma_{i}:=[0,2 \pi] \times\left\{r_{i}\right\}, \\ v_{\epsilon}=0 & \text { on } \Gamma_{e}:=[0,2 \pi] \times\left\{r_{e}\right\} \\ \int_{\Omega} v_{\epsilon}^{2}=1, & \end{cases}
$$

and

$$
\begin{cases}\left(\frac{\partial^{2}}{\partial r^{2}}+\frac{1}{r} \frac{\partial}{\partial r}+\frac{1}{r^{2}} \frac{\partial^{2}}{\partial \theta^{2}}+\omega_{0}^{2}\right) v_{0}=0 & \text { in } \Omega \\ -\frac{\partial v_{0}}{\partial r}=0 & \text { on } \Gamma_{i} \\ v_{0}=0 & \text { on } \Gamma_{e} \\ \int_{\Omega} v_{0}^{2}=1 . & \end{cases}
$$


We solve these equations using the finite difference method. To do this, we discretize the equations at the node points on $\Omega$ given by

$$
\left(\theta_{n}, r_{m}\right)=\left(2 \pi \frac{n-1}{N}, r_{i}+\left(r_{e}-r_{i}\right) \frac{m}{M+1}\right)
$$

for $n=1,2, \cdots, N$, and $m=1,2, \cdots, M$, with $N=128, M=16$. Using the first eigenvalue and eigenvector computed, we solve (3.1) using the following $h$,

$$
h_{k}(\theta)=a_{0}+a_{1} \sin \theta+a_{2} \sin 2 \theta+\cdots+a_{k} \sin k \theta, \quad \theta \in \Gamma_{e},
$$

for $k=1,2, \ldots, 10$. Based on (3.5), the location $z$ of the corrosion is determined as the minimum point of the function

$$
J(z):=\sum_{i=1}^{10}\left|\frac{w_{h_{i}}(z)}{v_{0}(z)}-\frac{1}{2 \omega_{0}\left(\omega_{\epsilon}-\omega_{0}\right)} \int_{\Gamma_{e}} \frac{\partial v_{\epsilon}}{\partial \nu} h_{i}-\int_{\Omega} v_{0} w_{h_{i}}\right| .
$$

We then compute $\gamma \epsilon$ using (3.6).

Examples 1, 2, 3 show the results of numerical experiments with various $\gamma$ and some noise added to the data. They clearly demonstrate the viability of our reconstruction approach.

Example 1. We implement the reconstruction method for two-dimensional disks using Matlab and finite difference method. $U$ and $D$ are the disks centered $(0,0)$ of radii 0.2 and 0.1 , respectively, and the corrosion coefficient is set to be 2 . Table 1 and Figure 1 summarize the computational results. The first figure shows the actual domain where the red part is the corrosion. The second figure is the graph of the function $J$ whose minimal point is the detected center of corrosion. The figures in the right-hand side are the eigenvectors with and without corrosion, $v_{\epsilon}$ and $v_{0}$. The errors are $\left|z_{s}-z_{s}^{c}\right|=0$ and $\left|\gamma \epsilon-(\gamma \epsilon)^{c}\right|=0.0035$ where $z_{s}^{c}$ and $(\gamma \epsilon)^{c}$ are the detected location of the corrosion and the corrosion coefficient.

Example 2. In this example, $U$ and $D$ are the disks centered $(0,0)$ of radii 1.0 and 0.8 , respectively. We test the algorithm with various corrosion coefficients $\gamma=0.01,2,5$, while the size of the corrosion is fixed at $\epsilon \approx 0.04$. We also add $1 \%, 5 \%, 10 \%$ noise when we compute the eigenvectors. It turns out that larger the corrosion coefficient is, better is the performance, which is quite natural. The results also show that our algorithm works fairly well even in the presence of noise provided that the corrosion coefficient is large enough. See Figure 2.

Example 3. This example provides the results of numerical tests with larger size of the corrosive part, $\epsilon \approx 0.15$. The results show that the algorithm works equally as well, or even better, in detecting the location of the corrosion. However, its performance in detecting $\gamma \epsilon$ is poorer than in the case of shorter corrosion. See Figure 3.

\section{Justification of the Asymptotic Expansion}

In this section we review the main results of Gohberg and Sigal in [8] and give a rigorous proof of Theorem 2.1 which was driven formally. 

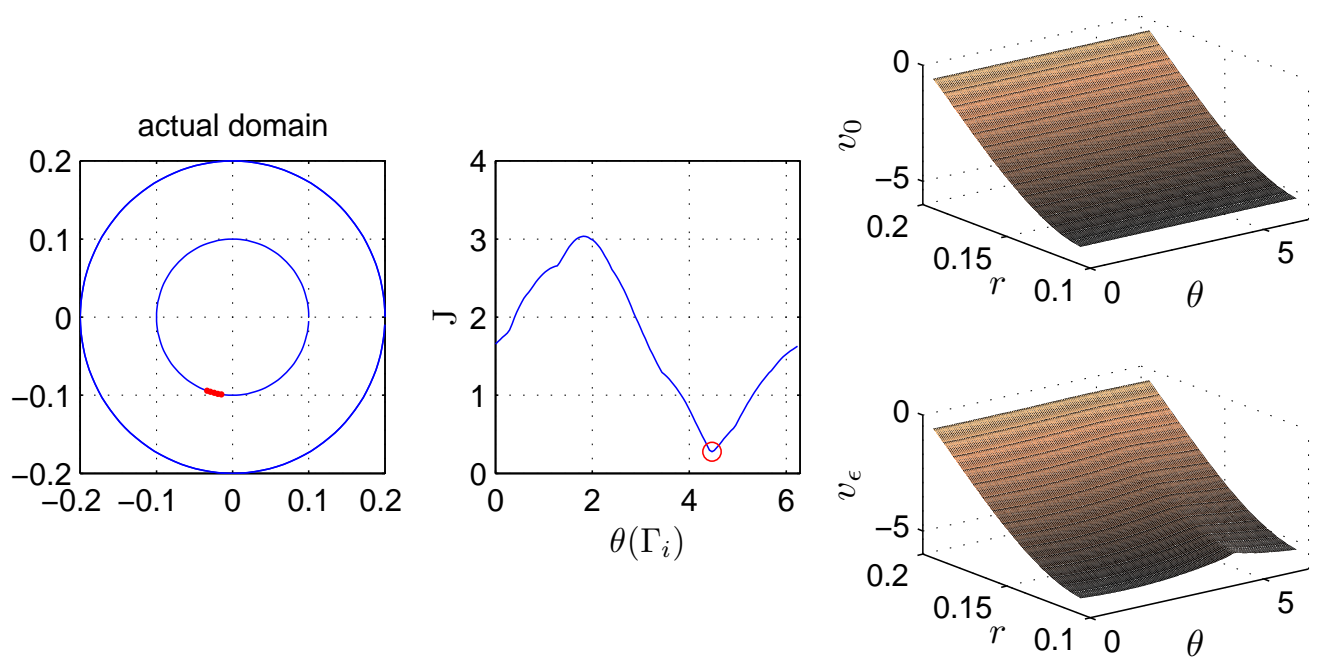

\begin{tabular}{cccc}
\hline$z_{s}$ & $z_{s}^{c}$ & $\gamma \epsilon$ & $(\gamma \epsilon)^{c}$ \\
\hline \hline$(-0.0243,-0.0970)$ & $(-0.0243,-0.0970)$ & 0.0393 & 0.0428 \\
\hline
\end{tabular}

Figure 1: Reconstruction result without noise. $z_{s}$ and $\gamma \epsilon$ are actual location and coefficients of the corrosion and $z_{s}^{c}$ and $(\gamma \epsilon)^{c}$ are detected ones.

Let $\mathcal{G}$ and $\mathcal{H}$ be two Banach spaces and let $\mathcal{L}(\mathcal{G}, \mathcal{H})$ be the set of all bounded operators from $\mathcal{G}$ to $\mathcal{H}$. Let $U$ be an open set in $\mathbb{C}$. Suppose that $A(\omega)$ is an operator-valued function from $U$ to $\mathcal{L}(\mathcal{G}, \mathcal{H}) . \omega_{0}$ is a characteristic value of $A(\omega)$ if

- $A(\omega)$ is holomorphic in some neighborhood of $\omega_{0}$, except possibly for $\omega_{0}$;

- There exists a function $\phi(\omega)$ from a neighborhood of $\omega_{0}$ to $\mathcal{G}$, holomorphic and nonzero at $\omega_{0}$ such that $A(\omega) \phi(\omega)$ is holomorphic at $\omega_{0}$ and $A\left(\omega_{0}\right) \phi\left(\omega_{0}\right)=0$.

The function $\phi(\omega)$ in the above definition is called a root function of $A(\omega)$ associated to $\omega_{0}$ and $\phi\left(\omega_{0}\right)$ is called an eigenvector. The closure of the space of eigenvectors corresponding to $\omega_{0}$ is denoted by $\operatorname{Ker} A\left(\omega_{0}\right)$.

Let $\phi_{0}$ be an eigenvector corresponding to $\omega_{0}$. Let $V\left(\omega_{0}\right)$ be a complex neighborhhod of $\omega_{0}$. The rank of $\phi_{0}$ is the largest integer $m$ such that there exist $\phi: V\left(\omega_{0}\right) \rightarrow \mathcal{G}$ and $\psi: V\left(\omega_{0}\right) \rightarrow \mathcal{H}$ holomorphic satisfying

$$
\begin{aligned}
& A(\omega) \phi(\omega)=\left(\omega-\omega_{0}\right)^{m} \psi(\omega), \\
& \phi\left(\omega_{0}\right)=\phi_{0}, \quad \psi\left(\omega_{0}\right) \neq 0 .
\end{aligned}
$$

Suppose that $n=\operatorname{dim} \operatorname{Ker} A\left(\omega_{0}\right)<+\infty$ and the ranks of all vectors in $\operatorname{Ker} A\left(\omega_{0}\right)$ are finite. A system of eigenvectors $\phi_{0}^{j}, j=1, \cdots, n$, is called a canonical system of eigenvectors of $A(\omega)$ associated to $\omega_{0}$ if the rank of $\phi_{0}^{j}$ is the maximum of the ranks of all eigenvectors in some direct complement in $\operatorname{Ker} A\left(\omega_{0}\right)$ of the linear span of the vectors $\phi_{0}^{1}, \cdots, \phi_{0}^{j-1}$. Then 
we define the null multiplicity of the characteristic value $\omega_{0}$ of $A(\omega)$ to be the sum of ranks of $\phi_{0}^{j}, j=1, \cdots, n$, which is denoted by $N\left(A\left(\omega_{0}\right)\right)$.

Suppose that $A^{-1}(\omega)$ exists and is holomorphic in some neighborhood of $\omega_{0}$, except possibly at this point itself. Then the number

$$
M\left(A\left(\omega_{0}\right)\right)=N\left(A\left(\omega_{0}\right)\right)-N\left(A^{-1}\left(\omega_{0}\right)\right)
$$

is called the multiplicity of the characteristic value $\omega_{0}$ of $A(\omega)$.

If $A(\omega)$ is holomorphic at $\omega_{0}$ and $A\left(\omega_{0}\right)$ is invertible, the point $\omega_{0}$ is called a regular point of $A(\omega)$. A point $\omega_{0}$ is called a normal point of $A(\omega)$ if there exists some neighborhood $V\left(\omega_{0}\right)$ of $\omega_{0}$ in which all the points except $\omega_{0}$ are regular points of $A(\omega)$ and $A(\omega)$ admits the Laurent expansion

$$
A(\omega)=\sum_{j \geq-s}\left(\omega-\omega_{0}\right)^{j} A_{j} .
$$

where the operators $A_{j}, j=-s, \cdots,-1$, are finite dimensional and the operator $A_{0}$ is a Fredholm operator. An operator-valued function $A(\omega)$ is called normal with respect to $\partial V\left(\omega_{0}\right)$ if $A(\omega)$ is holomorphic and invertible in $\overline{V\left(\omega_{0}\right)}$, except for a finite number of points of $V\left(\omega_{0}\right)$ which are normal points of $A(\omega)$.

Suppose that $A(\omega)$ is normal with respect to $\partial V\left(\omega_{0}\right)$ and $\omega_{i}, i=1, \cdots, \sigma$, are all its characteristic values and poles lying in $V\left(\omega_{0}\right)$, we put

$$
\mathcal{M}\left(A(\omega) ; \partial V\left(\omega_{0}\right)\right)=\sum_{i=1}^{\sigma} M\left(A\left(\omega_{i}\right)\right) .
$$

The generalization of Rouché's theorem is stated below.

Theorem 5.1 Let $A(\omega)$ be an operator-valued function which is normal with respect to $\partial V\left(\omega_{0}\right)$. If an operator-valued function $S(\omega)$ which is holomorphic in $V\left(\omega_{0}\right)$ and continuous at $\partial V\left(\omega_{0}\right)$ satisfies the condition

$$
\left\|A^{-1}(\omega) S(\omega)\right\|_{\mathcal{L}(\mathcal{G}, \mathcal{G})}<1, \quad \omega \in \partial V\left(\omega_{0}\right),
$$

then $A(\omega)+S(\omega)$ is also normal with respect to $\partial V\left(\omega_{0}\right)$ and

$$
\mathcal{M}\left(A(\omega) ; \partial V\left(\omega_{0}\right)\right)=\mathcal{M}\left(A(\omega)+S(\omega) ; \partial V\left(\omega_{0}\right)\right) .
$$

The generalization of the residue theorem is given by

Theorem 5.2 Suppose that $A(\omega)$ is an operator-valued function which is normal with respect to $\partial V\left(\omega_{0}\right)$. Let $f(\omega)$ be a scalar function which is holomorphic in $V\left(\omega_{0}\right)$ and continuous in $\overline{V\left(\omega_{0}\right)}$. Then we have

$$
\frac{1}{2 \pi i} \operatorname{tr} \int_{\partial V\left(\omega_{0}\right)} f(\omega) A^{-1}(\omega) \frac{d}{d \omega} A(\omega) d \omega=\sum_{j=1}^{\sigma} M\left(A\left(\omega_{j}\right)\right) f\left(\omega_{j}\right),
$$

where $\omega_{j}, j=1, \cdots, \sigma$, are all the points in $V\left(\omega_{0}\right)$ which are either poles or characteristic values of $A(\omega)$. 
Here $\operatorname{tr}$ denotes the trace of operator which is the sum of all its nonzero eigenvalues.

A fundamental solution $\Phi_{\omega}(x)$ to the Helmholtz operator $\Delta+\omega^{2}$ in $\mathbb{R}^{2}$ is given by

$$
\Phi_{\omega}(x)=-\frac{i}{4} H_{0}^{(1)}(\omega|x|),
$$

for $x \neq 0$, where $H_{0}^{(1)}$ is the Hankel function of the first kind of order 0 . Let $\Gamma$ be either $\Gamma_{i}$ or $\Gamma_{e}$, and define $\mathcal{S}_{\Gamma}^{\omega}$ and $\mathcal{D}_{\Gamma}^{\omega}$ be the single and double layer potentials defined by $\Phi_{\omega}$, that is,

$$
\begin{aligned}
& \mathcal{S}_{\Gamma}^{\omega}[\varphi](x)=\int_{\Gamma} \Phi_{\omega}(x-y) \varphi(y) d \sigma(y), \quad x \in \mathbb{R}^{2}, \\
& \mathcal{D}_{\Gamma}^{\omega}[\varphi](x)=\int_{\Gamma} \frac{\partial \Phi_{\omega}(x-y)}{\partial \nu_{y}} \varphi(y) d \sigma(y), \quad x \in \mathbb{R}^{2} \backslash \Gamma,
\end{aligned}
$$

for $\varphi \in L^{2}(\Gamma)$.

The following formulae give the jump relations obeyed by the double layer potential and by the normal derivative of the single layer potential:

$$
\begin{gathered}
\left.\left(\mathcal{D}_{\Gamma}^{\omega}[\varphi]\right)\right|_{ \pm}(x)=\left(\mp \frac{1}{2} I+\mathcal{K}_{\Gamma}^{\omega}\right)[\varphi](x) \quad \text { a.e. } x \in \Gamma \\
\left.\frac{\partial\left(\mathcal{S}_{\Gamma}^{\omega}[\varphi]\right)}{\partial \nu}\right|_{ \pm}(x)=\left( \pm \frac{1}{2} I+\left(\mathcal{K}_{\Gamma}^{\omega}\right)^{*}\right)[\varphi](x) \quad \text { a.e. } x \in \Gamma
\end{gathered}
$$

for $\varphi \in L^{2}(\Gamma)$, where $\mathcal{K}_{\Gamma}^{\omega}$ is the operator defined by

$$
\mathcal{K}_{\Gamma}^{\omega}[\varphi](x)=\text { p.v. } \int_{\Gamma} \frac{\partial \Phi_{\omega}(x-y)}{\partial \nu_{y}} \varphi(y) d \sigma(y),
$$

and $\left(\mathcal{K}_{\Gamma}^{\omega}\right)^{*}$ is the $L^{2}$-adjoint of $\mathcal{K}_{\Gamma}^{\omega}$, that is,

$$
\left(\mathcal{K}_{\Gamma}^{\omega}\right)^{*}[\varphi](x)=\text { p.v. } \int_{\Gamma} \frac{\partial \Phi_{\omega}(x-y)}{\partial \nu_{x}} \varphi(y) d \sigma(y) .
$$

Here p.v. stands for the Cauchy principal value. The singular integral operators $\mathcal{K}_{\Gamma}^{\omega}$ and $\left(\mathcal{K}_{\Gamma}^{\omega}\right)^{*}$ are known to be bounded on $L^{2}(\Gamma)$. Here and throughout this paper the subscripts \pm as in (5.2) denote the limits from outside and inside of $\Gamma$.

In order to investigate the eigenvalues of the problem (2.2), we consider the operator $\mathbf{A}_{0}^{\omega}: L^{2}\left(\Gamma_{e}\right) \times H^{1}\left(\Gamma_{i}\right) \rightarrow H^{1}\left(\Gamma_{e}\right) \times H^{1}\left(\Gamma_{i}\right)$ defined by

$$
\mathbf{A}_{0}^{\omega}:=\left(\begin{array}{cc}
\mathcal{S}_{\Gamma_{e}}^{\omega} & \mathcal{D}_{\Gamma_{i}}^{\omega} \\
\mathcal{S}_{\Gamma_{e}}^{\omega} & \frac{1}{2} I+\mathcal{K}_{\Gamma_{i}}^{\omega}
\end{array}\right) .
$$

Here $H^{1}\left(\Gamma_{e}\right)$ denotes the set of functions $f \in L^{2}\left(\Gamma_{e}\right)$ such that $\partial f / \partial T \in L^{2}\left(\Gamma_{e}\right)$, where $\partial / \partial T$ is the tangential derivative. $H^{1}\left(\Gamma_{i}\right)$ is defined likewise.

Observe that $\omega \mapsto \mathbf{A}_{0}^{\omega}$ is an operator valued holomorphic function. The relation between the eigenvalues of (2.2) and the characteristic values of $\mathbf{A}_{0}^{\omega}$ is given by the following theorem. 
Theorem 5.3 Suppose that $-\omega^{2}$ is not a Dirichlet eigenvalue of $\Delta$ on D. Then, $-\omega^{2}$ is an eigenvalue of (2.2) if and only if $\omega$ is a characteristic value of $\mathbf{A}_{0}^{\omega}$.

Proof. Suppose that $\omega^{2}$ is an eigenvalue of (2.2) so that there is a nontrivial solution $v$ to (2.2). Then by Green's representation formula we have

$$
v(x)=-\mathcal{S}_{\Gamma_{e}}^{\omega}\left[\left.\frac{\partial v}{\partial \nu}\right|_{\Gamma_{e}}\right](x)-\mathcal{D}_{\Gamma_{i}}^{\omega}\left[\left.v\right|_{\Gamma_{i}}\right](x), \quad x \in \Omega
$$

Put $\varphi:=\left.\frac{\partial v}{\partial \nu}\right|_{\Gamma_{e}}$ and $\psi:=\left.v\right|_{\Gamma_{i}}$. Then $(\varphi, \psi) \in L^{2}\left(\Gamma_{e}\right) \times H^{1}\left(\Gamma_{i}\right)$ is not zero and satisfies

$$
\mathcal{S}_{\Gamma_{e}}^{\omega}[\varphi]+\mathcal{D}_{\Gamma_{i}}^{\omega}[\psi]=0 \quad \text { on } \Gamma_{e}
$$

On the other hand, by (5.2), we have

$$
\left.\left(\mathcal{S}_{\Gamma_{e}}^{\omega}[\varphi]+\mathcal{D}_{\Gamma_{i}}^{\omega}[\psi]\right)\right|_{+}-\left.\left(\mathcal{S}_{\Gamma_{e}}^{\omega}[\varphi]+\mathcal{D}_{\Gamma_{i}}^{\omega}[\psi]\right)\right|_{-}=-\psi=-\left.v\right|_{\Gamma_{i}} \quad \text { on } \Gamma_{i},
$$

and hence $\left.\left(\mathcal{S}_{\Gamma_{e}}^{\omega}[\varphi]+\mathcal{D}_{\Gamma_{i}}^{\omega}[\psi]\right)\right|_{-}=0$ on $\Gamma_{i}$, or equivalently,

$$
\mathcal{S}_{\Gamma_{e}}^{\omega}[\varphi]+\left(\frac{1}{2} I+\mathcal{K}_{\Gamma_{i}}^{\omega}\right)[\psi]=0 \quad \text { on } \Gamma_{i} .
$$

Combining (5.4) and (5.5) shows that $\omega$ is a characteristic value of $A_{0}^{\omega}$.

Conversely, suppose that $w$ is a characteristic value of $\mathbf{A}_{0}^{\omega}$ so that there is a non-zero $(\varphi, \psi) \in L^{2}\left(\Gamma_{e}\right) \times H^{1}\left(\Gamma_{i}\right)$ satisfying

$$
\mathbf{A}_{0}^{\omega}\left(\begin{array}{c}
\varphi \\
\psi
\end{array}\right)=0
$$

or equivalently (5.4) and (5.5). Define

$$
v(x):=-\mathcal{S}_{\Gamma_{e}}^{\omega}[\varphi](x)-\mathcal{D}_{\Gamma_{i}}^{\omega}[\psi](x), \quad x \in \Omega .
$$

Then $v=0$ on $\Gamma_{e}$ by (5.4). On the other hand, (5.5) shows that $\psi \in \mathcal{C}^{1, \alpha}\left(\Gamma_{i}\right)$ for some $\alpha>0$. In fact, by (5.5), we have

$$
\psi=2 \mathcal{S}_{\Gamma_{e}}^{\omega}[\varphi]-2 \mathcal{K}_{\Gamma_{i}}^{\omega}[\psi] .
$$

Since $\Gamma_{i}$ is $\mathcal{C}^{2}, \mathcal{K}_{\Gamma_{i}}^{\omega}$ maps $L^{2}\left(\Gamma_{i}\right)$ into $L^{\infty}\left(\Gamma_{i}\right), L^{\infty}\left(\Gamma_{i}\right)$ into $\mathcal{C}^{\alpha}(\Gamma)$ for all $\alpha<1$, and $\mathcal{C}^{\alpha}(\Gamma)$ into $\mathcal{C}^{1, \alpha}(\Gamma)$. Thus by bootstrapping using (5.8), we have $\psi \in \mathcal{C}^{1, \alpha}\left(\Gamma_{i}\right)$. Now $\frac{\partial}{\partial \nu} \mathcal{D}_{\Gamma_{i}}^{\omega}[\psi]$ is well-defined and it does not have a jump along $\Gamma_{i}$, i.e.,

$$
\left.\frac{\partial}{\partial \nu} \mathcal{D}_{\Gamma_{i}}^{\omega}[\psi]\right|_{+}=\left.\frac{\partial}{\partial \nu} \mathcal{D}_{\Gamma_{i}}^{\omega}[\psi]\right|_{-} \text {on } \Gamma_{i}
$$

Since $\omega^{2}$ is not a Dirichlet eigenvalue of $-\Delta$ on $D,(5.5)$ implies that $\mathcal{S}_{\Gamma_{e}}^{\omega}[\varphi]+\mathcal{D}_{\Gamma_{i}}^{\omega}[\psi]=0$ in $D$, and hence

$$
\left.\frac{\partial}{\partial \nu}\left(\mathcal{S}_{\Gamma_{e}}^{\omega}[\varphi]+\mathcal{D}_{\Gamma_{i}}^{\omega}[\psi]\right)\right|_{-}=0 \quad \text { on } \Gamma_{i}
$$


We thus obtain

$$
\begin{aligned}
\left.\frac{\partial v}{\partial \nu}\right|_{\Gamma_{i}} & =\left.\frac{\partial}{\partial \nu}\left(\mathcal{S}_{\Gamma_{e}}^{\omega}[\varphi]+\mathcal{D}_{\Gamma_{i}}^{\omega}[\psi]\right)\right|_{+} \\
& =\left.\frac{\partial}{\partial \nu}\left(\mathcal{S}_{\Gamma_{e}}^{\omega}[\varphi]+\mathcal{D}_{\Gamma_{i}}^{\omega}[\psi]\right)\right|_{-}=0
\end{aligned}
$$

In other words, $v$ is an eigenfunction of the problem (2.2). This completes the proof.

In a similar way, one can prove the following theorem for the problem (2.1).

Theorem 5.4 Define the operator $\mathbf{A}_{\epsilon}^{\omega}: L^{2}\left(\Gamma_{e}\right) \times H^{1}\left(\Gamma_{i}\right) \rightarrow H^{1}\left(\Gamma_{e}\right) \times H^{1}\left(\Gamma_{i}\right)$ by

$$
\mathbf{A}_{\epsilon}^{\omega}:=\left(\begin{array}{cc}
\mathcal{S}_{\Gamma_{e}}^{\omega} & \mathcal{D}_{\Gamma_{i}}^{\omega}-\mathcal{S}_{\Gamma_{i}}^{\omega} M_{\epsilon} \\
\mathcal{S}_{\Gamma_{e}}^{\omega} & \frac{1}{2} I+\mathcal{K}_{\Gamma_{i}}^{\omega}-\mathcal{S}_{\Gamma_{i}}^{\omega} M_{\epsilon}
\end{array}\right)
$$

where $M_{\epsilon}$ means the multiplication by $\gamma \chi(I)$. Assume that $-\omega^{2}$ is not a Dirichlet eigenvalue of $\Delta$ on D. Then $-\omega^{2}$ is an eigenvalue of (2.1) if and only if $\omega$ is a characteristic value of $\mathbf{A}_{\epsilon}^{\omega}$.

Observe that we can write

$$
\mathbf{A}_{\epsilon}^{\omega}=\mathbf{A}_{0}^{\omega}+\epsilon \mathbf{B}_{\epsilon}^{\omega}
$$

where

$$
\mathbf{B}_{\epsilon}^{\omega}:=\left(\begin{array}{cc}
0 & -\frac{1}{\epsilon} \mathcal{S}_{\Gamma_{i}}^{\omega} M_{\epsilon} \\
0 & -\frac{1}{\epsilon} \mathcal{S}_{\Gamma_{i}}^{\omega} M_{\epsilon}
\end{array}\right) .
$$

Since $M_{\epsilon}$ is of order $\epsilon$, the operator $\mathbf{B}_{\epsilon}^{\omega}$ is of order 1 .

Lemma $5.5 \mathbf{A}_{0}^{\omega}$ is a Fredholm operator of index 0 and every eigenvector of $\mathbf{A}_{0}^{\omega}$ has rank one provided that $-\omega_{0}^{2}$ is not a Dirichlet eigenvalue of $\Delta$ on $D$.

Proof. Since, written in the following manner, $\mathbf{A}_{0}^{\omega}$ is clearly a compact perturbation of Fredholm operator of index 0

$$
\mathbf{A}_{0}^{\omega}=\left(\begin{array}{cc}
\mathcal{S}_{\Gamma_{e}}^{\omega} & 0 \\
0 & \frac{1}{2} I+\mathcal{K}_{\Gamma_{i}}^{\omega}
\end{array}\right)+\left(\begin{array}{cc}
0 & \mathcal{D}_{\Gamma_{i}}^{\omega} \\
\mathcal{S}_{\Gamma_{e}}^{\omega} & 0
\end{array}\right)
$$

hence it is Fredholm of index 0.

Suppose that $\left(\begin{array}{l}\varphi \\ \psi\end{array}\right)$ is an eigenvector of $\mathbf{A}_{0}^{\omega}$ with rank $m$ associated with the characteristic value $\omega_{0}$, i.e., there exist $\varphi^{\omega}$ and $\psi^{\omega}$, holomorphic as functions of $\omega$, such that $\varphi^{\omega_{0}}=\varphi$, $\psi^{\omega_{0}}=\psi$, and

$$
\mathbf{A}_{0}^{\omega}\left(\begin{array}{l}
\varphi^{\omega} \\
\psi^{\omega}
\end{array}\right)=\left(\omega-\omega_{0}\right)^{m}\left(\begin{array}{c}
\widetilde{\varphi}^{\omega} \\
\widetilde{\psi}^{\omega}
\end{array}\right)
$$


for some $\left(\widetilde{\varphi}^{\omega}, \widetilde{\psi}^{\omega}\right) \in H^{1}\left(\Gamma_{e}\right) \times H^{1}\left(\Gamma_{i}\right)$. Let $u^{\omega}:=\mathcal{S}_{\Gamma_{e}}^{\omega}\left[\varphi^{\omega}\right]+\mathcal{D}_{\Gamma_{i}}^{\omega}\left[\psi^{\omega}\right]$. Then because of (5.11), $u^{\omega}$ satisfies

$$
\begin{cases}\left(\Delta+\omega^{2}\right) u^{\omega}=0 & \text { in } \mathbb{R}^{2} \backslash\left(\Gamma_{e} \cup \Gamma_{i}\right), \\ u^{\omega}=\left(\omega-\omega_{0}\right)^{m} \widetilde{\varphi}^{\omega} & \text { on } \Gamma_{e}, \\ \left.u^{\omega}\right|_{-}=\left(\omega-\omega_{0}\right)^{m} \widetilde{\psi}^{\omega} & \text { on } \Gamma_{i} .\end{cases}
$$

Since $-\omega_{0}^{2}$ is not a Dirichlet eigenvalue of $\Delta$ on $D, \mathcal{S}_{\Gamma_{i}}^{\omega}: L^{2}\left(\Gamma_{i}\right) \rightarrow H^{1}\left(\Gamma_{i}\right)$ is invertible for $\omega$ in a neighborhood of $\omega_{0}$, and hence we have

$$
u^{\omega}(x)=\left(\omega-\omega_{0}\right)^{m} \mathcal{S}_{\Gamma_{i}}^{\omega}\left[\left(\mathcal{S}_{\Gamma_{i}}^{\omega}\right)^{-1} \widetilde{\psi}^{\omega}\right](x), \quad x \in D,
$$

and

$$
\left.\frac{\partial u^{\omega}}{\partial \nu}\right|_{+}=\left.\frac{\partial u^{\omega}}{\partial \nu}\right|_{-}=\left(\omega-\omega_{0}\right)^{m}\left(-\frac{1}{2} I+\left(\mathcal{K}_{\Gamma_{i}}^{\omega}\right)^{*}\right)\left[\left(\mathcal{S}_{\Gamma_{i}}^{\omega}\right)^{-1} \widetilde{\psi}^{\omega}\right] \quad \text { on } \Gamma_{i} .
$$

By Green's formula, we immediately get

$$
\begin{aligned}
& \left(\omega^{2}-\omega_{0}^{2}\right) \int_{\Omega} u^{\omega} u^{\omega_{0}} \\
& =\int_{\Gamma_{e}} \frac{\partial u^{\omega_{0}}}{\partial \nu} u^{\omega}+\int_{\Gamma_{i}} \frac{\partial u^{\omega}}{\partial \nu} u^{\omega_{0}} \\
& =\left(\omega-\omega_{0}\right)^{m}\left(\int_{\Gamma_{e}} \frac{\partial u^{\omega_{0}}}{\partial \nu} \widetilde{\varphi}^{\omega}+\int_{\Gamma_{i}} u^{\omega_{0}}\left(-\frac{1}{2} I+\left(\mathcal{K}_{\Gamma_{i}}^{\omega}\right)^{*}\right)\left[\left(\mathcal{S}_{\Gamma_{i}}^{\omega}\right)^{-1} \widetilde{\psi}^{\omega}\right]\right) .
\end{aligned}
$$

If $m>1$, by dividing both sides by $\omega^{2}-\omega_{0}^{2}$ and taking the limit as $\omega \rightarrow \omega_{0}$, we obtain $\int_{\Omega}\left(u^{\omega_{0}}\right)^{2}=0$ which is a contradiction. Thus we have $m=1$. This completes the proof.

By the above lemma and the generalized Rouché's theorem (Theorem 5.1), we know that $\mathbf{A}_{\epsilon}^{\omega}$ is normal with respect to a small neighborhood $V$ of $\omega_{0}$ and that the multiplicity of $\mathbf{A}_{\epsilon}^{\omega}$ in $V$ is equal to the dimension of the eigenspace of (2.2) associated with $\omega_{0}$. Now we are ready to prove Theorem 2.1 .

The following lemma was proved in [3]. We include a proof for the readers' sake.

Lemma 5.6 Let $V$ be a small neighborhood of $\omega_{0}$ in a complex plane such that $\mathbf{A}_{\epsilon}^{\omega}$ has the simple characteristic value $\omega_{\epsilon}$ in $V$. Then

$$
\omega_{\epsilon}-\omega_{0}=\frac{1}{2 \pi i} \sum_{j=1}^{\infty} \frac{(-1)^{j} \epsilon^{j}}{j} \operatorname{tr} \int_{\partial V}\left(\left(\mathbf{A}_{0}^{\omega}\right)^{-1} \mathbf{B}_{\epsilon}^{\omega}\right)^{j} d \omega .
$$

Proof. It follows from Theorem 5.2 and Lemma 5.5 that

$$
\omega_{\epsilon}-\omega_{0}=\frac{1}{2 \pi i} \operatorname{tr} \int_{\partial V}\left(\omega-\omega_{0}\right)\left(\mathbf{A}_{\epsilon}^{\omega}\right)^{-1} \frac{d}{d \omega} \mathbf{A}_{\epsilon}^{\omega} d \omega
$$

By (5.9), one can see that

$$
\left(\mathbf{A}_{\epsilon}^{\omega}\right)^{-1}=\sum_{j=0}^{\infty}(-1)^{j} \epsilon^{j}\left(\left(\mathbf{A}_{0}^{\omega}\right)^{-1} \mathbf{B}_{\epsilon}^{\omega}\right)^{j}\left(\mathbf{A}_{0}^{\omega}\right)^{-1},
$$


where the series converges in the operator norm on $L^{2}\left(\Gamma_{e}\right) \times H^{1}\left(\Gamma_{i}\right) \rightarrow H^{1}\left(\Gamma_{e}\right) \times H^{1}\left(\Gamma_{i}\right)$ if $\epsilon$ is sufficiently small. If we substitute (5.14) into (5.13), we have

$$
\omega_{\epsilon}-\omega_{0}=\frac{1}{2 \pi i} \sum_{j=0}^{\infty}(-1)^{j} \epsilon^{j} \operatorname{tr} \int_{\partial V}\left(\omega-\omega_{0}\right)\left(\left(\mathbf{A}_{0}^{\omega}\right)^{-1} \mathbf{B}_{\epsilon}^{\omega}\right)^{j}\left(\mathbf{A}_{0}^{\omega}\right)^{-1} \frac{d}{d \omega} \mathbf{A}_{\epsilon}^{\omega} d \omega .
$$

Since

we have

$$
\frac{d}{d \omega}\left(\mathbf{A}_{0}^{\omega}\right)^{-1}=-\left(\mathbf{A}_{0}^{\omega}\right)^{-1} \frac{d}{d \omega} \mathbf{A}_{0}^{\omega} \cdot\left(\mathbf{A}_{0}^{\omega}\right)^{-1},
$$

$$
\begin{aligned}
& \epsilon \frac{d}{d \omega}\left(\left(\mathbf{A}_{0}^{\omega}\right)^{-1} \mathbf{B}_{\epsilon}^{\omega}\right)^{j}=j\left(\left(\mathbf{A}_{0}^{\omega}\right)^{-1} \mathbf{B}_{\epsilon}^{\omega}\right)^{j-1}\left(\mathbf{A}_{0}^{\omega}\right)^{-1} \frac{d}{d \omega} \mathbf{A}_{\epsilon}^{\omega} \\
& -\epsilon j\left(\left(\mathbf{A}_{0}^{\omega}\right)^{-1} \mathbf{B}_{\epsilon}^{\omega}\right)^{j}\left(\mathbf{A}_{0}^{\omega}\right)^{-1} \frac{d}{d \omega} \mathbf{A}_{0}^{\omega}-j\left(\left(\mathbf{A}_{0}^{\omega}\right)^{-1} \mathbf{B}_{\epsilon}^{\omega}\right)^{j-1}\left(\mathbf{A}_{0}^{\omega}\right)^{-1} \frac{d}{d \omega} \mathbf{A}_{0}^{\omega} .
\end{aligned}
$$

We now substitute (5.16) into (5.15). Then the sum of the last two terms in (5.16) cancel each other and hence we have

$$
\omega_{\epsilon}-\omega_{0}=-\frac{1}{2 \pi i} \sum_{j=1}^{\infty} \frac{(-1)^{j} \epsilon^{j}}{j} \operatorname{tr} \int_{\partial V}\left(\omega-\omega_{0}\right) \frac{d}{d \omega}\left(\left(\mathbf{A}_{0}^{\omega}\right)^{-1} \mathbf{B}_{\epsilon}^{\omega}\right)^{j} d \omega .
$$

Now (5.12) immediately follows and the proof is complete.

Proof of Theorem 2.1. Let

$$
\left(\mathbf{A}_{0}^{\omega}\right)^{-1}=\left(\begin{array}{cc}
A_{1}^{\omega} & A_{2}^{\omega} \\
A_{3}^{\omega} & A_{4}^{\omega}
\end{array}\right)
$$

Then we have

$$
-\frac{\epsilon}{2 \pi i} \operatorname{tr} \int_{\partial V}\left(\mathbf{A}_{0}^{\omega}\right)^{-1} \mathbf{B}_{\epsilon}^{\omega} d \omega=\frac{1}{2 \pi i} \operatorname{tr} \int_{\partial V}\left(A_{3}^{\omega} \mathcal{S}_{\Gamma_{i}}^{\omega} M_{\epsilon}+A_{4}^{\omega} \mathcal{S}_{\Gamma_{i}}^{\omega} M_{\epsilon}\right) d \omega .
$$

Let $0<\mu_{1} \leq \mu_{2} \leq \ldots$ be the eigenvalues of $(2.2)$ and $u_{1}, u_{2}, \ldots$ be the corresponding normalized orthogonal eigenfunctions. For $\phi \in L^{2}\left(\Gamma_{i}\right)$, let

$$
u:=-\mathcal{S}_{\Gamma_{e}}^{\omega}\left(A_{1}^{\omega} \mathcal{S}_{\Gamma_{i}}^{\omega}+A_{2}^{\omega} \mathcal{S}_{\Gamma_{i}}^{\omega}\right)[\phi]-\mathcal{D}_{\Gamma_{i}}^{\omega}\left(A_{3}^{\omega} \mathcal{S}_{\Gamma_{i}}^{\omega}+A_{4}^{\omega} \mathcal{S}_{\Gamma_{i}}^{\omega}\right)[\phi]+\mathcal{S}_{\Gamma_{i}}^{\omega}[\phi] .
$$

Then, by the definition of $A_{i}^{\omega}$ 's, $u$ satisfies

$$
\begin{cases}\left(\Delta+\omega^{2}\right) u=0 & \text { in } \Omega, \\ \frac{\partial u}{\partial \nu}=\phi & \text { on } \Gamma_{i}, \\ u=\left(A_{3}^{\omega} \mathcal{S}_{\Gamma_{i}}^{\omega}+A_{4}^{\omega} \mathcal{S}_{\Gamma_{i}}^{\omega}\right)[\phi] & \text { on } \Gamma_{i}, \\ u=0 & \text { on } \Gamma_{e} .\end{cases}
$$

Applying Green's formula, we have

$$
\left(\omega^{2}-\mu_{i}\right) \int_{\Omega} u u_{i}=\int_{\Gamma_{i}} \phi u_{i}
$$


and hence

$$
u=\sum_{n=1}^{\infty} \frac{\left\langle u_{i}, \phi\right\rangle_{\Gamma_{i}}}{\omega^{2}-\mu_{i}} u_{i} \quad \text { in } \Omega
$$

where $\langle\cdot, \cdot\rangle_{\Gamma_{i}}$ denotes the inner product in $L^{2}\left(\Gamma_{i}\right)$. By taking the trace on $\Gamma_{i}$, we obtain

$$
\left(A_{3}^{\omega} \mathcal{S}_{\Gamma_{i}}^{\omega}+A_{4}^{\omega} \mathcal{S}_{\Gamma_{i}}^{\omega}\right)[\phi]=\left.\sum_{n=1}^{\infty} \frac{\left\langle u_{i}, \phi\right\rangle_{\Gamma_{i}}}{\omega^{2}-\mu_{i}} u_{i}\right|_{\Gamma_{i}}
$$

Then we have

$$
\begin{aligned}
\frac{1}{2 \pi i} \operatorname{tr} \int_{\partial V}\left(A_{3}^{\omega} \mathcal{S}_{\Gamma_{i}}^{\omega} M_{\epsilon}+A_{4}^{\omega} \mathcal{S}_{\Gamma_{i}}^{\omega} M_{\epsilon}\right) d \omega & =\frac{1}{2 \pi i} \int_{\partial V} \sum_{n=1}^{\infty} \frac{\left\langle u_{i}, \gamma \chi(I) u_{i}\right\rangle_{\Gamma_{i}}}{\omega^{2}-\mu_{i}} d \omega \\
& =\frac{\gamma}{2 \omega_{0}} \int_{I} v_{0}^{2}
\end{aligned}
$$

since $\omega_{0}^{2}$ is the only eigenvalue inside $V$. Therefore

$$
\omega_{\epsilon}-\omega_{0}=\frac{\gamma}{2 \omega_{0}} \int_{I} v_{0}^{2}+O\left(\epsilon^{2}\right)
$$

This proves $(2.7)$

We now prove the (2.8). Choose $\left(\begin{array}{c}\varphi_{\epsilon} \\ \psi_{\epsilon}\end{array}\right) \in \operatorname{Ker} A_{\epsilon}^{\omega_{\epsilon}}$. Let $\Psi_{\epsilon}=\left(\begin{array}{l}\varphi_{\epsilon} \\ \psi_{\epsilon}\end{array}\right)$ for convenience and assume that $\left\|\Psi_{\epsilon}\right\|_{L^{2}\left(\Gamma_{e}\right) \times H^{1}\left(\Gamma_{i}\right)}=1$. Let us define $P_{\epsilon}$ by

$$
P_{\epsilon}=\frac{1}{2 \pi i} \int_{\partial V}\left(\mathbf{A}_{\epsilon}^{\omega}\right)^{-1} \frac{d}{d \omega} \mathbf{A}_{\epsilon}^{\omega} d \omega .
$$

Then it is proved in [8] that $P_{\epsilon}$ is a projection (not necessarily orthogonal) from $L^{2}\left(\Gamma_{e}\right) \times$ $H^{1}\left(\Gamma_{i}\right)$ onto $\operatorname{Ker} A_{\epsilon}^{\omega_{\epsilon}}$. It follows from (5.14) that

$$
P_{\epsilon}=P_{0}+O(\epsilon)
$$

where $O(\epsilon)$ is in the operator norm. Having both sides of (5.18) act on $\Psi_{\epsilon}$, we obtain

$$
\Psi_{\epsilon}=P_{0} \Psi_{\epsilon}+O(\epsilon)
$$

where $O(\epsilon)$ is in $L^{2}\left(\Gamma_{e}\right) \times H^{1}\left(\Gamma_{i}\right)$-norm. Let $P_{0} \Psi_{\epsilon}=\left(\varphi_{\epsilon}^{0}, \psi_{\epsilon}^{0}\right)$ and

$$
v_{\epsilon}=\mathcal{S}_{\Gamma_{e}}^{\omega_{\epsilon}}\left[\varphi_{\epsilon}\right]+\mathcal{D}_{\Gamma_{i}}^{\omega_{\epsilon}}\left[\psi_{\epsilon}\right]-\mathcal{S}_{\Gamma_{i}}^{\omega_{\epsilon}} M_{\epsilon}\left[\psi_{\epsilon}\right], \quad v_{0}=\mathcal{S}_{\Gamma_{e}}^{\omega_{0}}\left[\varphi_{\epsilon}^{0}\right]+\mathcal{D}_{\Gamma_{i}}^{\omega_{0}}\left[\psi_{\epsilon}^{0}\right] \quad \text { in } \Omega .
$$

Since $\mathcal{S}_{\Gamma_{e}}^{\omega_{\epsilon}}$ and $\mathcal{D}_{\Gamma_{i}}^{\omega_{\epsilon}}$ map $L^{2}\left(\Gamma_{e}\right)$ and $H^{1}\left(\Gamma_{i}\right)$ into $H^{3 / 2}(\Omega)$, respectively, we have from (5.19) that

$$
v_{\epsilon}=v_{0}+O(\epsilon) \text { in } H^{3 / 2}(\Omega) .
$$

Now we normalize $v_{\epsilon}$ and $v_{0}$ by dividing by its $L^{2}(\Omega)$ norms and denote them by $v_{\epsilon}$ and $v_{0}$ again. Then $v_{\epsilon}, v_{0}$ are solutions of $(2.1),(2.2)$ respectively. Since $\left\|v_{\epsilon}\right\|_{L^{2}(\Omega)}=\left\|v_{0}\right\|_{L^{2}(\Omega)}+$ $O(\epsilon)$, they still satisfy (5.21). The proof of Theorem 2.1 is now complete. 


\section{Conclusion}

We have designed a simple and accurate method for detecting small internal corrosion by vibration analysis. Our method is based on asymptotic formulae for the resonance frequencies and mode shapes perturbations caused by internal corrosive parts of small Hausdorff measure.

To rigorously prove our asymptotic formulae we have reduced the problem to the study of the characteristic values of integral operators in the complex plane and made use of powerful techniques from the theory of meromorphic operator-valued functions.

We test the algorithm numerically on various situation and demonstrate its viability. It is worth noticing the fact that it is impossible to extract the size of the corrosive parts and the impedance coefficient using the first order approximation. We can only reconstruct the product of these two quantities. It is likely that from a certain level of signal-to-noise ratio, higher-order asymptotic expansions of the resonances and mode shapes perturbations yield such important information.

\section{References}

[1] H. Ammari and H. Kang, Reconstruction of Small Inhomogeneities from Boundary Measurements, Lecture Notes in Mathematics, Volume 1846, Springer-Verlag, Berlin, 2004.

[2] H. Ammari, H. Kang, E. Kim, K. Louati, and M. Vogelius, A MUSIC-type method for detecting internal corrosion from steady state voltage boundary perturbations, preprint.

[3] H. Ammari, H. Kang, M. Lim, and H. Zribi, Layer potential techniques in spectral analysis. Part I: Complete asymptotic expansions for eigenvalues of the Laplacian in domains with small inclusions, preprint.

[4] H.T. Banks, M.L. Joyner, B. Wincheski, and W.P. Winfree, Real time computational algorithms for eddy-current-based damage detection, Inverse Problems, 18 (2002), 795823.

[5] G. Buttazzo and R.V. Kohn, Reinforcement by a thin layer with oscillating thickness, Appl. Math. Opt., 16 (1988), 247-261.

[6] D.J. Cedio-Fengya, S. Moskow, and M.S. Vogelius, Identification of conductivity imperfections of small diameter by boundary measurements. Continuous dependence and computational reconstruction, Inverse Problems, 14 (1998), 553-595.

[7] A. Friedman and M.S. Vogelius, Identification of small inhomogeneities of extreme conductivity by boundary measurements: a theorem on continuous dependence, Arch. Rat. Mech. Anal., 105 (1989), 299-326.

[8] I.T.S. Gohberg and E.I. Sigal, Operator extension of the logarithmic residue theorem and Rouché's theorem, Mat. Sb. (N.S.) 84 (1971), 607-642.

[9] G. Inglese, An inverse problem in corrosion detection, Inverse Problems, 13 (1997), 977-994. 
[10] B. Luong and F. Santosa, Quantitative imaging of corrosion inplates by eddy current methods, SIAM J. Appl. Math., 58 (1998), 1509-1531.

[11] T. Kato, Perturbation Theory for Linear Operators, Springer-Verlag, New York, 1976.

[12] P. Kaup and F. Santosa, Nondestructive evaluation of corrosion damage using electrostatic measurements, J. Nondestructive Eval. 14 (1995), 127-136.

[13] P. Kaup, F. Santosa, and M. Vogelius, A method for imaging corrosion damage in thin plates from electrostatic data, Inverse Problems, 12 (1996), 279-293.

[14] O. Kwon, J.K. Seo, and J.R. Yoon, A real-time algorithm for the location search of discontinuous conductivities with one measurement, Comm. Pure Appl. Math., 55 (2002), 1-29.

[15] P.E. Mix, Introduction to Nondestructive Testing (Second Edition), Wiley, 2005.

[16] M. Vogelius and J. Xu, A nonlinear elliptic boundary value problem related to corrosion modelling, Quart. Appl. Math., 56 (1998), 479-505. 

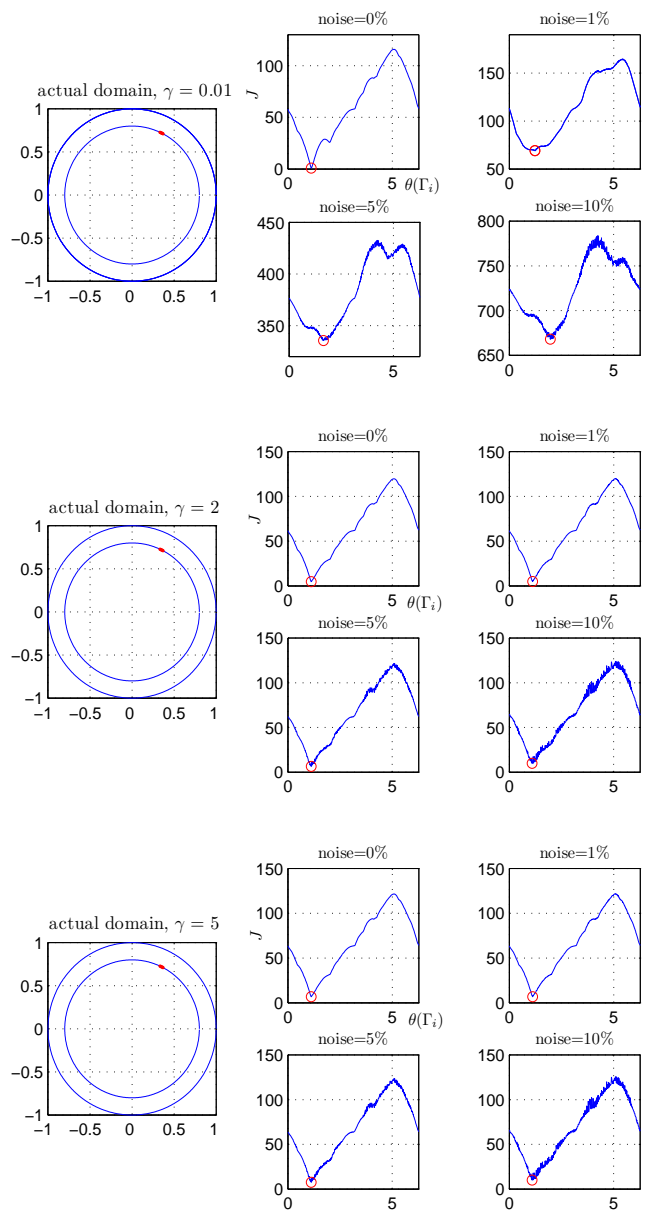

\begin{tabular}{cccccc}
\hline$\gamma$ & noise $(\%)$ & $z_{s}$ & $z_{s}^{c}$ & $\gamma \epsilon$ & $(\gamma \epsilon)^{c}$ \\
\hline 0.01 & 0 & $(0.3509,0.7189)$ & $(0.3509,0.7189)$ & 0.0004 & 0.0005 \\
0.01 & 1 & $(0.3509,0.7189)$ & $(0.2695,0.7532)$ & 0.0004 & 0.0005 \\
0.01 & 5 & $(0.3509,0.7189)$ & $(-0.0589,0.7978)$ & 0.0004 & 0.0005 \\
0.01 & 10 & $(0.3509,0.7189)$ & $(-0.3061,0.7391)$ & 0.0004 & 0.0006 \\
\hline 2 & 0 & $(0.3509,0.7189)$ & $(0.3509,0.7189)$ & 0.0785 & 0.0644 \\
2 & 1 & $(0.3509,0.7189)$ & $(0.3509,0.7189)$ & 0.0785 & 0.0656 \\
2 & 5 & $(0.3509,0.7189)$ & $(0.3509,0.7189)$ & 0.0785 & 0.0711 \\
2 & 10 & $(0.3509,0.7189)$ & $(0.3684,0.7101)$ & 0.0785 & 0.0717 \\
\hline 5 & 0 & $(0.3509,0.7189)$ & $(0.3509,0.7189)$ & 0.1963 & 0.1043 \\
5 & 1 & $(0.3509,0.7189)$ & $(0.3509,0.7189)$ & 0.1963 & 0.1063 \\
5 & 5 & $(0.3509,0.7189)$ & $(0.3509,0.7189)$ & 0.1963 & 0.1151 \\
5 & 10 & $(0.3509,0.7189)$ & $(0.36840 .7101)$ & 0.1963 & 0.1162 \\
\hline
\end{tabular}

Figure 2: $r_{i}=0.8, r_{e}=1$ 

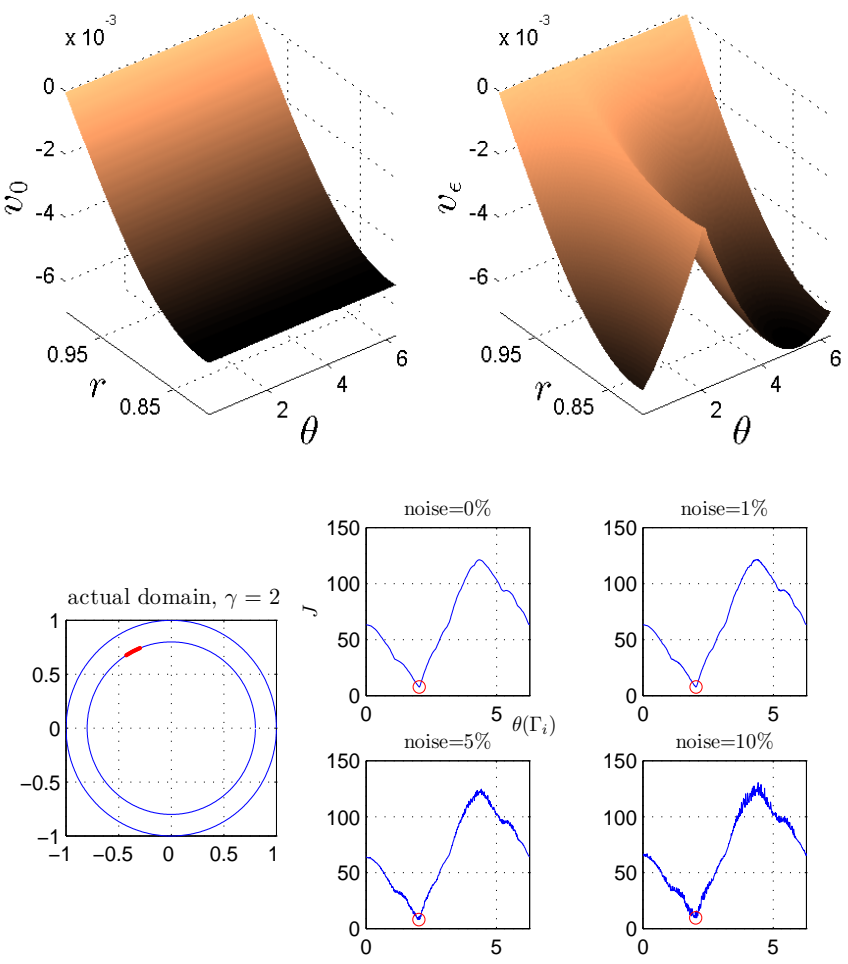

\begin{tabular}{cccccc}
\hline$\gamma$ & noise $(\%)$ & $z_{s}$ & $z_{s}^{c}$ & $\gamma \epsilon$ & $(\gamma \epsilon)^{c}$ \\
\hline 2 & 0 & $(-0.3684,0.7101)$ & $(-0.3597,0.7146)$ & 0.2945 & 0.1200 \\
2 & 1 & $(-0.3684,0.7101)$ & $(-0.3597,0.7146)$ & 0.2945 & 0.1199 \\
2 & 5 & $(-0.3684,0.7101)$ & $(-0.3509,0.7189)$ & 0.2945 & 0.1235 \\
2 & 10 & $(-0.3684,0.7101)$ & $(-0.3509,0.7189)$ & 0.2945 & 0.1271 \\
\hline
\end{tabular}

Figure 3: $r_{i}=0.8, r_{e}=1$ 